\title{
Opto-digital tomographic reconstruction of the Wigner distribution function of complex fields
}

\author{
Walter D. Furlan, ${ }^{1, \star}$ Carlos Soriano, ${ }^{2}$ and Genaro Saavedra ${ }^{1}$ \\ 'Departamento de Óptica, Universitat de València, E-46100 Burjassot (Valencia), Spain \\ ${ }^{2}$ Departamento de Procesos de Fabricación, Fundación Tekniker, E-20600 Eibar, Spain \\ ${ }^{*}$ Corresponding author: walter.furlan@uv.es
}

Received 3 January 2008; revised 6 May 2008; accepted 6 May 2008; posted 8 May 2008 (Doc. ID 91140); published 21 May 2008

\begin{abstract}
An optical-digital method has been developed to obtain the Wigner distribution function of onedimensional complex fields. In this technique an optical setup is employed to experimentally achieve the Radon-Wigner spectrum of the original signal through intensity measurements. Digital tomographic reconstruction is applied to the experimental spectrum to reconstruct the two-dimensional Wigner distribution function of the input. The validity of our proposal is demonstrated with experimental data, and the results are compared with computer simulations. (C) 2008 Optical Society of America

OCIS codes: $\quad 070.1170,100.6950$.
\end{abstract}

\section{Introduction}

The Wigner distribution function (WDF) is a phasespace representation that was introduced in optics more than 30 years ago [1]. Since then, it has found numerous applications and has become a very useful tool for the theoretical analysis of optical systems (see Ref. [2] and references therein). This formalism is characterized by the simple and elegant transformation rules it follows for describing the propagation of optical fields through linear systems and the graphical way in which they can be interpreted.

Since the WDF of a complex field distribution contains all the necessary information to retrieve the field itself $[3,4]$, several methods to obtain the WDF that have been proposed previously in the literature could be used to this end. Optical or optoelectronic devices are the most commonly employed systems to obtain a representation of WDFs of one-dimensional (1D) or two-dimensional (2D) complex signals [5-7]. However, because most detectors are sensitive to only the incident intensity, the optically obtained WDF, which is real but has in general negative values, is obtained with an uncertainty in

0003-6935/08/220E $63-05 \$ 15.00 / 0$

(C) 2008 Optical Society of America its sign. On the other hand, obtaining the WDF of wavefields is also possible indirectly through other representations such as the Radon transform (RT) [8]. In this particular case, a tomographic reconstruction is needed to synthesize the WDF and to recover the amplitude and the phase of the original field distribution solely by means of intensity measurements.

In all the experimental setups that have been tested for phase retrieval $[3,4]$, these measurements have been taken sequentially in time, varying the distances between some components for each single measure and losing in this way the potential advantage of parallel processing that optics may provide. In this paper, a new hybrid optodigital method to overcome this handicap is proposed. We use a simple optical device to obtain, in a single display (i.e., in parallel), the Radon-Wigner spectrum of an input 1D signal. The optical setup has no moving parts. Afterwards, the WDF of the original object is reconstructed tomographically by means of the inverse Radon transformation of the irradiance output of the optical system.

\section{Proposed Technique: Thoretical Background}

The main goal of this contribution is to obtain a representation of the WDF of a 1D complex field. If this field is represented by the function $f(x)$, its WDF is defined as 


$$
W_{f}(x, \omega)=\int_{-\infty}^{+\infty} f\left(x+x^{\prime} / 2\right) f^{*}\left(x-x^{\prime} / 2\right) e^{i 2 \pi \omega x^{\prime}} \mathrm{d} x^{\prime},
$$

where the symbol $*$ denotes complex conjugate. As stated in the Introduction section, several authors have proposed different techniques to obtain this transformation by optical means. Since in all cases the display of this function is achieved as the irradiance distribution in the output plane of the optical device, no information about the sign of the WDF is provided. In fact, it is well known that, except for the trivial case of Gaussian fields, all functions $f(x)$ provide both positive and negative values for their WDFs. This is a very critical issue, since quite different fields may provide equal values for $\left|W_{f}(x, \omega)\right|^{2}$. However there is a biunivocal relationship between every pair $\left(f(x), W_{f}(x, \omega)\right)$.

To overcome this incomplete reconstruction, we propose a new approach for obtaining the values of the WDF of a complex field from the experimental data recorded in a single $2 \mathrm{D}$ intensity measurement. In our proposal, instead of measuring the WDF itself, we take measurements of a complete set of projections of the WDF, which have the property of having only positive values [2]. Each one of these projections is known as the Radon-Wigner transform (RWT) of the original field $f(x)$. The subsequent application of digital tomographic reconstruction techniques will provide us with the required full WDF information.

Mathematically, the RWT of a 1D function $f(x)$ is defined as the path integral (i.e., a projection) of the 2D WDF of $f(x)$ performed over a straight line in the phase space []]. Explicitly,

$$
\begin{aligned}
R_{f}(u, \theta)= & \int_{-\infty}^{+\infty} W f(u \times \cos \theta-\omega \times \sin \theta, u \times \sin \theta \\
& +\omega \times \cos \theta) \mathrm{d} \omega \quad \forall \theta \in[0, \pi]
\end{aligned}
$$

$\theta$ being the projection angle for the WDF [in Eq. (2) we have used a new coordinate system $(u, \theta)$ in the phase space defined by the rotation of the system $(x, \omega)$ by the angle $\theta$ ]. The $2 \mathrm{D}$ continuous display of this function, for all the possible projection angles, is known as the Radon-Wigner spectrum of $f(x)$. In computerassisted tomography the Radon spectrum is also known as a sinogram. This name comes from the fact that the Radon transform of a single off-center point in the function to be reconstructed is a sinusoid.

The RWT of $f(x)$ can also be obtained indirectly from its fractional Fourier transform $(\operatorname{FrFT}), F_{f}(x, \theta)$, which is another integral transformation of $f(x)$ defined as

$$
F_{f}(u, \theta)=\int_{-\infty}^{+\infty} f(x) K(\theta ; u, x) \mathrm{d} x
$$

being

$$
\begin{aligned}
K(\theta ; u, x)= & \frac{\exp (i \theta / 2)}{\sqrt{i \sin \theta}} \exp \left\{i \pi \left(u^{2} \cot \theta-2 u x \csc \theta\right.\right. \\
& \left.+x^{2} \cot \theta\right\} .
\end{aligned}
$$

The kernel $K(\theta, u, x)$ reduces to $\delta(u-x)$ and to $\delta(u+x)$ when $\theta=0$ and $\theta=\pi$, respectively. This means that $F_{f}(u, 0)=f(u)$ and $F_{f}(u, \pi)=f(-u)$. On the other hand, when $\theta=\pi / 2$ or $\theta=-\pi / 2$ we have the Fourier transform and the inverse Fourier transform of $f(x)$ respectively. Many other properties of the FrFT can be found in Refs. [9] and [10].

The mathematical relationship between the RWT of $f(x)$ and its FrFT has been established in Ref. [11] and is given by

$$
R_{f}(u, \theta)=\left|F_{f}(u, \theta)\right|^{2} .
$$

The FrFT of a given input function can be obtained experimentally by means of several simple optical setups $[11,12]$. Based on these results, several experimental methods for obtaining the Radon-Wigner spectrum were also proposed [13-15]. However, to the best of our knowledge, the only one that has found practical applications [16-18] is the one reported in Ref. [14]. A new application of that method is proposed here to obtain the WDF of any complex signal from intensity measurements.

Based on Eqs. (2) and (3), the technique that we propose is divided into two basic stages, as sketched in Fig. 1. In the first one, provided the theoretical relationship between RWT and the FrFT, the experimental Radon-Wigner spectrum of the input function is obtained from a $2 \mathrm{D}$ single-shot intensity measurement. In the second step, the desired WDF is obtained by computation of the tomographic reconstruction of the WDF from the experimental result in the first step.

\section{Optical Achievement of the Radon-Wigner Spectrum}

For the optical achievement of the Radon-Wigner spectrum of a 1D function we used the setup proposed by Granieri et al. [14]. The optical device is very simple, consisting of only two bulk elements: a cylindrical lens and a varifocal lens. As is shown in Fig. 2, the 1D object is illuminated with a cylindrical wavefront. In this way, all the Fresnel diffraction patterns of the object are axially located within the region limited by the object and the illumination focus line. Due to the 1D character of these patterns, for

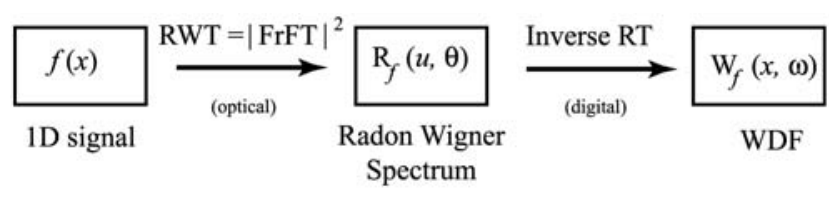

Fig. 1. Diagram of the proposed hybrid optodigital method. 


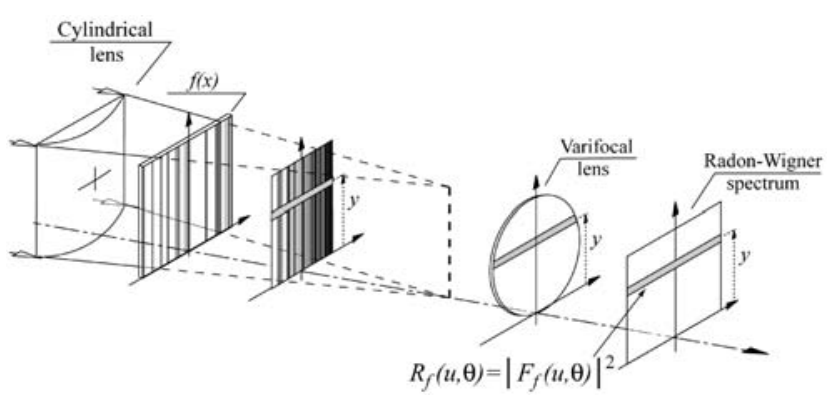

Fig. 2. Experimental device for obtaining the Radon-Wigner spectrum of a 1D object by means of intensity measurements. The cylindrical lens images all the diffraction patterns of the input object, each one of them located between the input and the focal line at a different axial position. The varifocal progressive lens images each and every one of the infinitesimal strips, located at different heights $(y)$ and each one corresponding to an individual pattern, at the same output plane.

each one of them it is possible to select $1 \mathrm{D}$ horizontal slices of infinitesimal width as independent information channels. The varifocal lens focuses simultaneously each one of these slices at different heights at the output plane. Provided the existing relationship between those diffraction patterns and the FrFT of the object [12], and using Eq. (3), it was demonstrated [9] that at the output plane one obtains a close approximation of the Radon-Wigner spectrum of the object. The Radon-Wigner spectrum obtained in this way comprises the angular region $\theta \in[0, \pi / 2]$. Note, however, that this is enough to complete the whole domain of the RWT (in which the $\theta$ parameter takes values from 0 to $\pi$ ) because of the symmetry property of the RWT, namely,

$$
R_{f}(u, \theta)=R_{f}(u,-\theta)=R_{f}(-u, \pi-\theta) .
$$

\section{Digital Tomographic Reconstruction of the WDF}

The second part of the proposed method is the digital computation of the inverse RTs of the experimental Radon-Wigner spectrum. The most common algorithms used in tomographic reconstruction are based on the technique known as filtered backprojection [19]. In this section we briefly present the steps that we followed to apply this technique in our reconstruction method.

The filtered backprojection algorithm is based on the central slice theorem, which states that the Fourier transform of the projection of a general function taken at an angle $\varphi$ equals the central slice, at the same angle, of the 2D Fourier transform of $g(x, y)$ [12]. In our case, the central slice theorem gives

$$
\tilde{R}_{f}(v, \theta)=\tilde{W}_{f}(\nu \cos \theta, \nu \sin \theta),
$$

where $\tilde{R}_{f}$ is the $1 \mathrm{D}$ Fourier transform of $R_{f}$ with respect to the spatial coordinate and $\tilde{W}_{f}$ is the $2 \mathrm{D}$ Fourier transform of $W_{f}$. The inversion of this last transformation allows the recovery of $W_{f}(x, \omega)$ from its projections

Explicitly,

$$
R_{f}(u, \theta)
$$

$$
\begin{aligned}
W_{f}(x, \omega)= & \int_{-\infty}^{+\infty} \int_{-\infty}^{+\infty} \tilde{W}_{f}(\xi, \psi) \exp \{2 \pi i(\xi x+\psi \omega\} \mathrm{d} \xi \mathrm{d} \psi \\
= & \int_{0}^{+\infty} \int_{0}^{\pi} \tilde{R}_{f}(v, \theta) \exp \{2 \pi i(x \times \cos \theta+\omega \\
& \times \sin \theta)\}|v| \mathrm{d} v \mathrm{~d} \theta
\end{aligned}
$$

where Eq. (7) has been properly used to change the integration variables. Finally, this result can be expressed as

$$
W_{f}(x, \omega)=\int_{0}^{\pi} S_{f}(x \times \cos \theta+\omega \times \sin \theta, \theta) \mathrm{d} \theta,
$$

being

$$
S_{f}(u, \theta)=\int_{-\infty}^{+\infty} \tilde{R}_{f}(v, \theta)|v| e^{2 i \pi v u} \mathrm{~d} v .
$$

Equation (10) can be clearly identified as a filtered version of the original RWT. In this way, from Eq. (9), $W_{f}(x, \omega)$ is reconstructed for each phasespace point $(x, \omega)$ as the superposition of all the projections $S_{f}(u, \theta)$ passing through this point.

\section{Results and Discussion}

The validity of our proposal has been verified with the experimental results obtained for the RadonWigner spectrum of different 1D functions. In Fig. 3 we show the optical displays obtained for two different functions, namely, a rectangular aperture (single slit) and a grating with a linearly increasing spatial frequency (chirp signal). These Radon-Wigner spectra were obtained with the optical device described in Fig. 2. It is interesting to note that the vertical slices of the continuous displays in Fig. 3 can be associated with the Fresnel diffraction patterns of the object evolving in the free space $[12,14]$.

It is worth to mention that to undertake the reconstruction of the WDF through the filtered backprojection algorithm it is necessary to consider the complete angular region of the Radon-Wigner spectrum, that is $\theta \in[0, \pi]$. Although we only obtain

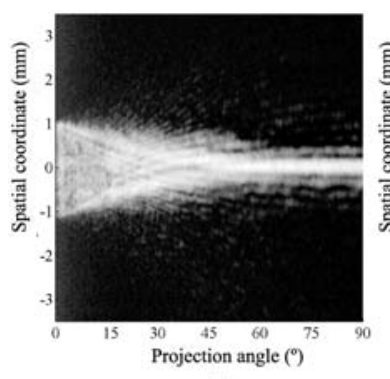

(a)

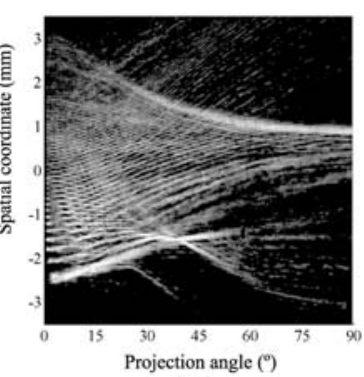

(b)
Fig. 3. Experimental Radon-Wigner spectrum of (a) a single slit of $2.2 \mathrm{~mm}$ and (b) a binary grating with a linearly increasing spatial frequency. 
optically the RWT for $\theta \in[0, \pi / 2]$ the symmetry property in Eq. (6) has been used to complete the spectrum. From the experimental Radon-Wigner spectra in Fig. 3, the corresponding WDFs have been obtained using the filtered backprojection algorithm.

For comparison purposes, Figs. 4 and 5 show both the theoretical and the experimentally reconstructed WDF of the single slit and the chirp grating. Note that in Figs. 4(b) and 5(b) some artifacts appear. The lines radiating from the center and outwards are artifacts (ringing effect) due to the filtered backprojection method [16]. In spite of this effect, a very good qualitative agreement can be observed between the results obtained with the theoretical and experimental data. It can be noted that the asymmetry in Fig. 4(b) is a consequence of the noise in Fig. 3(a), also reflecting the asymmetry on the spatial coordinate in this figure. From Fig. 5 the typical arrow-shaped WDF of a chirp function can be observed in both cases. It is noticeable that the slope in the arrow head that characterizes the chirp rate of the signal is the same for the theoretical and the experimental results.

\section{Conclusions}

A novel technique has been developed to obtain the WDF of a 1D signal. The method is divided into two steps. The first one implements a simple optical setup for obtaining the Radon-Wigner spectrum by means of a $2 \mathrm{D}$ single-shot intensity measurement. In the second step, this spectrum is used for a tomographic reconstruction of the WDF. Two different example objects, a single slit and a chirp signal, have been selected to prove the capabilities of the proposed method. The reconstructed WDF has been qualitatively compared with theoretical results, confirming the validity of the technique. Several extensions of the proposed method are straightforward. On the one hand, a similar implementation proposed here for the WDF can be easily derived for the ambiguity function, which is related to the WDF through a Fourier transformation [2]. Note also that it is easy to extend the method to obtain 2D samples of the 4D WDF of a complex $2 \mathrm{D}$ signal by use of a line scanning system. Moreover, since complex optical wavefields can be reconstructed from the WDF, provided the inversion formulas [1], this approach can be used as a phase retrieval method, becoming an alternative to the con-

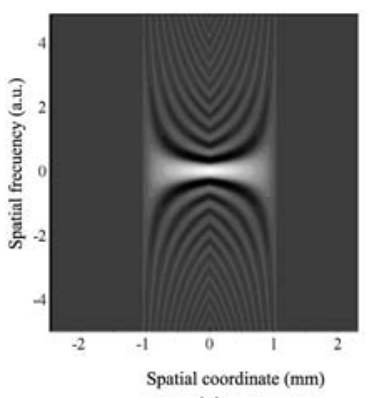

(a)

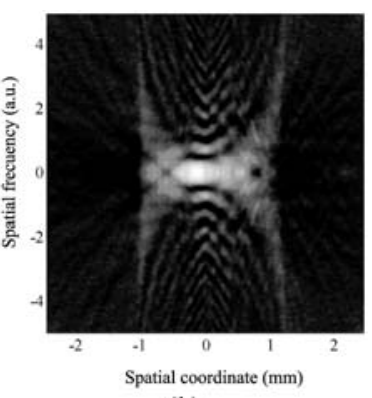

(b)
Fig. 4. (a) Theoretical WDF of a single slit. (b) Experimental result for the tomographic reconstruction of the WDF of the same slit.

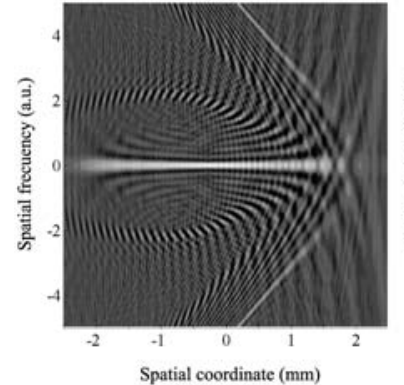

(a)

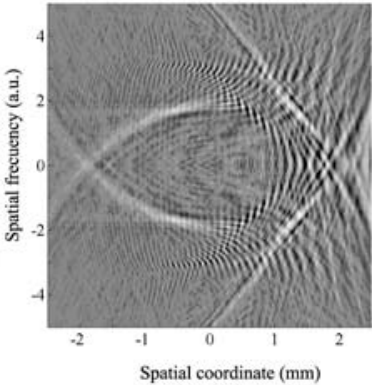

(b)
Fig. 5. (a) Theoretical WDF of a binary grating with a linearly increasing spatial frequency. (b) Experimental tomographic reconstruction of the WDF of the same grating.

ventional interferometric or iterative-algorithmbased techniques. In fact, as was very recently demonstrated [20], phase retrieval is possible with intensity measurements at two close FrFT domains, but this approach requires some $a$ priori knowledge of the signal bandwidth. In our method a continuous set of FrFTs is available simultaneously, and this redundancy should avoid any previous hypothesis about the input signal. This subject, and the improvement of the efficiency of the tomographic reconstruction (by taking advantage of certain general symmetries of the WDF), are current topics of our research program.

This research was supported by the Ministerio de Ciencia y Tecnología, Spain. grant DPI 2006-8309.

\section{References}

1. M. J. Bastiaans, "The Wigner distribution function applied to optical signals and systems," Opt. Commun. 25, 26-30 (1978).

2. D. Dragoman, "The Wigner distribution function in optics and optoelectronics,” Prog. Opt. 37, 3-53 (1997).

3. M. G. Raymer, M. Beck, and D. F. McAlister, "Complex wavefield reconstruction using phase-space tomography," Phys. Rev. Lett. 72, 1137-1140 (1994).

4. D. F. McAlister, M. Beck, L. Clarke, A. Mayer, and M. G. Raymer, "Optical phase retrieval by phase-space tomography and fractional-order Fourier transforms," Opt. Lett. 20, 1181-1183 (1994).

5. K. H. Brenner and A. W. Lohmann, "Wigner distribution function display of complex 1D signals," Opt. Commun. 42, 310314 (1982)

6. Y. Li, G. Eichmann, and M. Conner, "Optical Wigner distribution and ambiguity function for complex signals and images," Opt. Commun. 67, 177-179 (1988).

7. G. Shabtay, D. Mendlovic, and Z. Zalevsky, "Proposal for optical implementation of the Wigner distribution function," Appl. Opt. 37, 2142-2144 (1998).

8. R. L. Easton, Jr., A. J. Ticknor, and H. H. Barrett, "Application of the Radon transform to optical production of the Wigner distribution," Opt. Eng. 23, 738-744 (1984).

9. V. Namias, "The fractional order Fourier transform and its application to quantum mechanics," J. Inst. Appl. Math. 25, 241265 (1980)

10. L. B. Almeida, "The fractional Fourier transform and timefrequency representations," IEEE Trans. Signal Process. 42, 3084-3091 (1994).

11. A. W. Lohmann and B. H. Soffer, "Relationships between the Radon-Wigner and fractional Fourier transforms," J. Opt. Soc. Am. A 11, 1798-1801 (1994). 
12. P. Andrés, W. D. Furlan, G. Saavedra, and A. W. Lohmann, "Variable fractional Fourier processor: a simple implementation," J. Opt. Soc. Am. A 14, 853-858 (1997).

13. D. Mendlovic, R. G. Dorsch, A. W. Lohmann, Z. Zalevsky, and C. Ferreira, "Optical illustration of a varied fractional Fourier transform order and the Radon-Wigner chart," Appl. Opt. 35, 3925-3929 (1996).

14. S. Granieri, W. D. Furlan, G. Saavedra, and P. Andres, "Radon-Wigner display: a compact optical implementation with a single varifocal lens," Appl. Opt. 36, 8363-8369 (1997).

15. Y. Zhang, B. Gu, B. Dong, and G. Yang, "Optical implementations of the Radon-Wigner display for one-dimensional signals," Opt. Lett. 23, 1126-1128 (1998).
16. S. Granieri, E. E. Sicre, and W. D. Furlan, "Performance analysis of optical imaging systems based on the fractional Fourier transform," J. Mod. Opt. 45, 1797-1807 (1998).

17. O. Trabocchi, S. Granieri, and W. D. Furlan, "Optical propagation of fractal fields. Experimental analysis in a single display," J. Mod. Opt. 48, 1247-1253 (2001).

18. S. Granieri, M. Tebaldi, and W. D. Furlan, "Parallel fractional correlation: An optical implementation," Appl. Opt. 40, 64396444 (2001).

19. A. C. Kak and M. Slaney, Principles of Computerized Tomographic Imaging (IEEE Press, 1988).

20. U. Gopinathan, G. Situ, T. J. Naughton, and J. T. Sheridan, "Noninterferometric phase retrieval using a fractional Fourier system," J. Opt. Soc. Am. A 25, 108-115 (2008). 